\title{
Screening for Odorant Receptor Genes Expressed in Aedes Aegypti Involved in Blood-Feeding and Oviposition Behaviors
}

\section{Meng Ni}

Anhui Medical University

Teng Zhao ( $\square$ zhaoteng2013@163.com )

Academy of Military Medical Sciences State Key Laboratory of Pathogen and Biosecurity https://orcid.org/0000-0003-2143-3350

\section{Hui-xin Lv}

Academy of Military Medical Sciences State Key Laboratory of Pathogen and Biosecurity

\section{Man-jin Li}

Academy of Military Medical Sciences State Key Laboratory of Pathogen and Biosecurity

\section{Dan Xing}

Academy of Military Medical Sciences State Key Laboratory of Pathogen and Biosecurity

\section{Tong-yan Zhao}

Academy of Military Medical Sciences State Key Laboratory of Pathogen and Biosecurity

Chun-xiao Li

Academy of Military Medical Sciences State Key Laboratory of Pathogen and Biosecurity

\section{Research}

Keywords: Aedes aegypti, olfaction, odor receptor (OR), blood sucking, oviposition

Posted Date: December 29th, 2021

DOI: https://doi.org/10.21203/rs.3.rs-1168501/v1

License: (a) (1) This work is licensed under a Creative Commons Attribution 4.0 International License. Read Full License 


\section{Abstract}

Background: Aedes aegypti is one of the most important vector worldwide, and its survival and reproductive processes depend heavily on the olfactory system. In this study, the expression levels of all odorant receptor (OR) genes of Ae. aegypti were explored in different physiological periods to identify olfactory genes that may be associated with mosquito blood sucking and searching for oviposition sites.

Methods: Four groups, consisting of Ae. aegypti males (M), pre-blood-feeding females (F), post-bloodfeeding females (B) and post-oviposition females (O), were established. A total of 114 pairs of primer targeting all OR genes were designed based on the whole genome of Ae. aegypti. The expression of OR genes was evaluated by real-time fluorescence quantitative PCR for relative quantification and the comparison of differences between groups.

Results: A total of 53 differentially expressed OR genes were identified between males and females in $A e$. aegypti antennae. And 8, 5 and 13 differentially expressed OR genes were identified before versus after blood feeding, before versus after oviposition and post-blood-feeding versus post-oviposition, respectively. Meanwhile, 16 OR genes were significantly differentially expressed in multiple physiological periods of mosquitoes.

Conclusions: A large number of ORs with significant intergroup differences and high expression levels were screened in this study, including OR75, OR88, OR110 and OR115 and so on. Some of these genes are reported for the first time, providing possible targets for the development of mosquito control pathways based on the olfactory system.

\section{Background}

Aedes aegypti mosquitoes transmit diseases such as dengue fever, Zika, yellow fever and chikungunya, infecting hundreds of millions of people each year[1]. After mating, the female Ae. aegypti mainly use smell and temperature to locate the host and suck blood[2]. Mosquitoes mainly rely on their sensitive olfactory system to search for their hosts and lay eggs. In recent years, research on the olfactory genes of mosquitoes and the development of new biological control methods have gradually attracted attention[3,4].

The olfactory system in mosquitoes consists of odorant-binding proteins (OBPs), odorant receptors (ORs) and olfactory neurons (odorant receptor neurons OSNs). When odor molecules from the environment bind to ORs on the dendritic membrane of insect olfactory sensory neurons with the help of OBPs, the neurons are activated to convert external chemical stimuli into electrical signals[5], which trigger a series of subsequent behaviors. In 1999, Clyne[6] and Vosshall[7] discovered the first OR protein in Drosophila melanogaster. Different species of mosquitoes have different numbers of corresponding ORs. The numbers of OR genes identified in Anopheles gambiae[8], Ae. aegypt[[9], Culex quinquefasciatus[10] and Ae. albopictus[11] are 79, 131, 180 and 158, respectively. The OR genes have been identified in the genomes of two species of Aedes, 19 species of Anopheles and one species of Culex, and the list is 
growing[12]. The ORs are divided into two classes: traditional ligand-based ORs (ORx)[13] and coexpressing odorant receptors (odorant receptor coreceptors, Orcos), which are highly conserved in different insect species[14,15]. The high conservation and widespread expression of Orco genes across species suggest that Orcos may be an important component of olfactory function. While the spatiotemporal expression profiles and functions of traditional ORs are highly variable and species specific, the expression of different ORs in different mosquitoes is closely related to specific mosquito olfactory-mediated behaviors (host search and host preference)[16]. The ORs may recognize different types of odors or may be specific to a single odor[17,18]. For example, female An. gambiae mosquitoes specifically express AgOR1, which has a host-seeking function[19].

At present, some studies have been carried out on the OR gene of Ae. aegypti, and the correlation between certain OR and blood-sucking olfactory behavior has also been explored. The level of AaOR4 expression in Ae. aegypti affected its preference for human hosts[20]. The AaOR2 responded to indole, whch is the main component of human sweat[21]. The AaOR8 was more sensitive to (R)-1-octen-3-ol than to (S)-1octen-3-ol[22]. When the AaOR8 and AaOR49 (normally expressed in the stalk) were downregulated, the mosquitoes show an increase in stalk-probing behavior and longer blood sucking times, implying that these ORs are involved in the sucking process[23]. In this study, a systematic study of OR genes of Ae. aegypti was conducted to explore the OR genes that may influence host localization and oviposition in Ae. aegypti.

\section{Materials And Methods}

\section{Mosquitoes}

The Ae. aegypti strain is a laboratory strain, which was originally from Jinghong, Yunnan Province and has been kept in the laboratory for six years. The mosquitoes were reared at $26 \pm 1^{\circ} \mathrm{C}, 75 \pm 5 \%$ relative humidity, and a light:dark schedule of $14 \mathrm{~h}: 10 \mathrm{~h}$. The adult mosquitoes from the three strains were fed with $8 \%$ sugar water, $3-5$ days after emergence, fed with blood meal to breed the next generation.

Four groups of Ae. aegypti, consisting of males (M), pre-blood-feeding females (F), post-blood-feeding females (B) and post-oviposition females ( $O$ ), were conducted. The mosquitoes of group $M$ and $F$ are the adult mosquitoes that 3-5 $d$ after emergence. The mosquitoes of group $M$ and $F$ are the females at 1-2 $d$ after a blood meal. The mosquitoes of group 0 are the females after oviposition. Each group is to take 10 pairs of antennae of mosquitoes. Three biological replicates were set up in each group.

\section{Extraction and reverse transcription of total RNA}

Total RNA was extracted from groups $M, F, B$ and $O$ using the RNeasy MiNiKit reagent (QIAGEN) according to the reagent use instructions, and the concentration of total RNA was measured by UV spectrophotometry. The Prime ScriptR RTase kit (TAKARA) was employed according to the provided instructions. The reaction conditions were as follows: $37^{\circ} \mathrm{C}$ for $30 \mathrm{~min}, 85^{\circ} \mathrm{C}$ for $5 \mathrm{sec}$, and $-20^{\circ} \mathrm{C}$ for storage on standby. 


\section{Primer design and synthesis}

The Ae. aegypti OR gene sequence (GCF_002204515.2) was downloaded from NCBI (https://www.ncbi.nlm.nih.gov/), and 114 pairs of primers were designed using Primer premier 5.0, which were then synthesized by Tianyi Huiyuan and are listed in Additional file 1: Dataset S1.

\section{Real-time fluorescence quantitative polynucleotide chain reaction (qRT-PCR) analysis of OR gene expression}

The obtained mosquito antennae cDNA was employed as a template for real-time fluorescent quantitative PCR using the PerfectStart Green qPCR SuperMix assay kit from TransGen Biotech. The quantification of each sample was repeated three times, and three replicates were performed for each group. Reaction procedure: $94{ }^{\circ} \mathrm{C}$ for $30 \mathrm{sec}$, followed by 40 cycles of $94{ }^{\circ} \mathrm{C}$ for $5 \mathrm{sec}$, and $60{ }^{\circ} \mathrm{C}$ for 34 sec. The real-time PCR amplification and lysis curves were confirmed after the reaction. rPS6 was used as the internal reference gene. The applied primer sequences were RPS6-F: 5'-CGTCGTCAGGAACGTATCC-3' and RPS6-R: 5'-TTCTTGGCAGCCTTAGCAG-3'.

\section{Statistical analysis}

The calculation of relative target gene expression levels was performed using the $2^{\wedge}{ }^{-} \Delta{ }^{\mathrm{Ct}}$ method (with rsp6 as an internal control). One-way ANOVA of the experimental data was performed using IBM SPSS Statistics 21 software, and multiple comparison analysis was performed when the overall differences were statistically significant. Dunnett T3 tests were used for multiple comparisons when the variance was not uniform, and post hoc Tukey's HSD tests were used for multiple comparisons when the variance was uniform. $\mathrm{P}<0.05$ was considered to indicate a statistically significant difference. Graphs were created using GraphPad Prism 8.3 and Tableau 2021.3 software.

\section{Results}

\section{Differentially expressed OR genes between male and female mosquitoes}

The qRT-PCR results showed that there were $47 \mathrm{Ae}$. aegypti OR genes with lower antennal expression in males than in females, including OR2, OR10, OR11 and OR13 and so on. Among these genes, OR2, OR4, OR6, OR71, OR115 and OR132 showed variable splicing. The expression of OR115-V2 and OR115-V3 in female mosquitoes was 103.02 and 109.48 times that in male mosquitoes, and the expression of OR88, OR107 and OR114 in female mosquitoes was $45.14,17.99$ and 21.10 times that in male mosquitoes, respectively. The expression of OR32, OR75, OR76, OR77, OR85 and OR89 was higher in male antennae than in female antennae, with expression differences of $6.65,2.63,2.45,4.23,2.26$ and 6.14 fold, respectively (Fig. 1). 


\section{Differentially expressed OR genes in female mosquitoes before and after blood sucking}

Eight OR genes were significantly differentially expressed in Ae. aegypti antennae before and after blood sucking $(\mathrm{P}<0.05)$. OR115-V2, OR115-V3, and OR116 were downregulated after blood feeding compared to their levels before blood feeding, with expression differences of 3.47, 3.15 and 3.11 fold, respectively. OR27, OR30, OR62, OR86, OR91 and OR117 were upregulated after blood feeding compared with their levels before blood feeding, with expression differences of 3.64, 2.54, 3.61, 4.55, 3.80 and 4.61 fold, respectively (Fig. 2).

\section{Differentially expressed OR genes in female mosquitoes before and after oviposition}

There were five OR genes that were expressed at lower levels in the antennae of post-oviposition females than in those of pre-oviposition females, including OR38, OR93, OR110, OR112, OR115-V2, and OR115-V3, with expression differences of 2.52, 4.17, 3.30, 2.10, 3.85 and 3.20 fold, respectively. There were three OR genes that were more highly expressed in the antennae of post-oviposition females than in those of preoviposition females, including OR75, OR77 and OR117, with expression differences of 3.04, 4.09 and 3.46 fold, respectively (Fig. 3).

\section{Differentially expressed olfactory genes in female mosquitoes after oviposition versus after blood feeding}

Ten OR genes were less highly expressed in the antennae of post-oviposition females than in those of blood-feeding females, including OR4-C, OR27, OR30, OR62, OR81, OR86, OR91, OR93, OR110 and OR119, with expression differences of 2.24, 3.58, 2.22, 3.51, 2.36, 3.09, 4.76, 5.97, 3.22 and 2.41 fold, respectively. Three OR genes were more highly expressed in the antennae of post-oviposition females than in those of blood-feeding females, including OR75, OR76 and OR77, with expression differences of 4.04, 2.28 and 3.53 fold, respectively (Fig. 4).

\section{OR genes with significantly different expression at different physiological periods of mosquitoes}

Multiple comparison analysis was performed based on the premise that the overall difference was statistically significant $(\mathrm{P}<0.05)$, and it was found that OR38 and OR112 were differentially expressed between male and female Ae. aegypti mosquitoes, with 3.16 and 5.55 fold higher expression in females than in males, respectively; their expression was also different before and after oviposition in females, with 2.52 and 2.10 fold higher expression in pre-oviposition females than in post-oviposition females, respectively. OR117 was differentially expressed in female mosquitoes before and after blood feeding, with 4.61 fold higher expression in blood-sucking females than in non-blood-sucking females This gene 
also differentially expressed in females before and after oviposition, with 3.46 fold higher expression in pre-oviposition females than in post-oviposition females. OR4-C, OR76 and OR119 were differentially expressed in Ae. aegypti mosquitoes between males and females, with 2.50, 0.41 and 5.46 fold higher expression in females than in males, respectively. The differences in the expression of these genes were also observed in the comparison between the post-oviposition and post-blood feeding groups of females, with 2.24, 0.44 and 2.41 fold higher expression in blood-feed females than in post-oviposition females, respectively. OR30 and OR86 were differentially expressed in the female mosquitoes before and after blood feeding. Their expression in blood-fed female mosquitoes was 2.54 and 4.55 fold higher than that in non-blood-sucking females, respectively. They were also differentially expressed between the postoviposition and post-blood-sucking groups. Their expression was 2.22 and 3.09 fold higher in bloodsucking female mosquitoes than in post-oviposition females, respectively (Fig. 5).

OR115-V2 and OR115-V3 were differentially expressed not only between males and females of Ae. aegypti and in females before and after blood feeding but also before and after oviposition. Their expression levels were 103.02 and 109.48 fold higher in females than in males, 3.47 and 3.15 fold higher in females before versus after blood feeding, and 3.85 and 3.20 fold higher in females before versus after oviposition, respectively. OR75, OR77 and OR110 were differentially expressed not only between males and females of Ae. aegypti and in females before versus after oviposition but also in females after oviposition versus after blood feeding, with $0.38,0.24$ and 3.42 fold higher expression in females than in males, $0.33,0.23$ and 3.30 fold higher expression in females before versus after oviposition, $0.25,0.28$ and 3.22 fold higher expression in females after blood feeding versus after oviposition, respectively. OR27, OR62 and OR91 were differentially expressed not only between males and females of Ae. aegypti and before and after blood feeding by females but also in the comparison between post-oviposition and post-feeding, with $3.11,9.40$ and 4.68 fold higher expression in females than in males, respectively; and 3.64, 3.61 and 3.80 fold higher in blood feeding females than in female, respectively; and 3.58, 3.51 and 4.76 fold higher in blood feeding females than in post-oviposition females, respectively (Fig. 6).

Detailed relative expression of OR gene in Ae. aegypti was shown on Additional file 2: Dataset S2.

\section{Discussion}

Ae. aegypti is one of the most important vector organisms in the world, and its survival and reproduction rely heavily on the olfactory system. As one of the key components of the olfactory system, ORs are mainly used to sense various chemical information in the environment. In this study, a systematic analysis of $114 \mathrm{OR}$ genes expression in male, non-blood-sucking females, blood-sucking females, and post-oviposition females of Ae. aegypti were performed, a large number of unreported OR genes that may be related to the host searching and oviposition in mosquitoes was found.

By comparing the expression levels of OR mRNAs in An. gambiae antennae, latrou[24] found that the expression of ORs was more easily activated by host odorants in females than in males. In this study, OR88, OR114, OR115-V2 and OR115-V3 in Ae. aegypti were found to be highly expressed in female 
antennae, and their expression levels differed significantly between male and female mosquitoes, suggesting that they may serve as key receptors for host odor detection in females. OR2 and OR4 have been confirmed to be related to the preference of mosquitoes for human hosts[20,21], and there are also differences between male and female in this study. At the same time, Matthews[25] found that OR84, OR87, OR88, OR100, OR103, OR104, OR105, OR112, OR113 by transcriptome sequencing are different between male and female mosquitoes. This study is consistent with the results.

Mosquitoes show reduced olfactory activity after blood sucking and shut down the olfactory response to control energy expenditure to allow midgut blood digestion and ovary development, and the reactivation of ORs is delayed after the end of blood digestion to allow females to search for egg-laying sites[26]. Based on the finding that OR115-V2, OR115-V3, and OR116 are highly expressed before blood sucking and show significantly decreased expression after blood sucking, it is hypothesized that these genes are involved in regulating blood sucking in mosquitoes. Among these genes, downregulated OR116 expression after blood sucking was also indicated by the sequencing results of Matthews[25].

When mosquitoes got enough blood, they need to find suitable oviposition sites to breed their offspring. Their perception of various oviposition attractants increases $24 \mathrm{~h}$ after blood sucking. The upregulation of OR expression may be related to a combination of oviposition lures that induce mosquitoes to find suitable oviposition sites[27]. A comprehensive analysis of the upregulated members of the chemosensory gene family among differentially expressed genes identified before and after a blood meal in An. sinensis by Chen[28]. revealed that they were associated with the precise odor recognition function in this mosquito. The qRT-PCR results showed that OR27, OR30, OR62, OR86, OR91 and OR117 were significantly upregulated after blood feeding, so these genes were presumed to be related to the search for suitable oviposition sites based on oviposition attractants by mosquitoes to breed their offspring. $\mathrm{Wu}[29]$ found that CquiOR114/117 was upregulated after blood feeding in $C x$. quinquefasciatus. The expression of ORs is regulated in mosquitoes according to their needs related to physiological functions. OR38, OR93, OR110, OR112, OR115-V2 and OR115-V3 are highly expressed before oviposition, and their expression decreases after oviposition, suggesting that they may play a role in mosquito oviposition in combination with oviposition stimulants. OR75, OR77 and OR117 are upregulated after oviposition, the mosquito enters the next blood-sucking and oviposition cycle after oviposition. The upregulation of expression may be involved in regulating the next blood-sucking and oviposition cycle of the mosquito.

There were at least two differences among the 16 ORs, represented by OR4, in the four groups of comparisons (male versus female, pre- versus post-blood-feeding females, pre- versus post-oviposition females, and post-blood-feeding females versus post-oviposition females). These results suggested a continuous role of ORs in all physiological period of mosquito to enable the use of infochemicals in the environment to locate mates, hosts, and oviposition sites throughout the mosquito's life cycle.

Highly homologous olfactory proteins in different mosquito species can respond to the same host odor and mosquito oviposition pheromones[30]. AgOR2 is highly conserved among mosquito species[17,21]. Cx. quinquefasciatus[31] and Ae. aegypti[21] show high OR2 homology, and both species respond to the 
oviposition attractant indole. Liu[32] simultaneously expressed AalOrco and AalOR10 of Ae. albopictus in human embryonic kidney cells and showed that they responded strongly to indole and 1-octen-3-ol, which are major components of human sweat. CquiOR10 and AsinOR10 recognize 3-methylindole volatilized at oviposition sites[33,34]. In this study, the expression of OR2 and OR10 in Ae. aegypti females was significantly higher than that in males was found, and the highest expression was found in females after blood feeding, These two genes are highly homologous to OR2 and OR10 in other species of mosquitoes. It is speculated that Ae. aegypti OR2 and OR10 may be involved in mosquito host localization and oviposition site-finding.

This study systematically and completely screened a large number of ORs that may affect the bloodsucking and oviposition behavior of Ae. aegypti mosquitoes, providing a direction forward in the analysis of the structures and interactions of these proteins. ORs with significant intergroup differences and high expression, such as OR93, OR110, OR115 and so on, provide possible targets for the development of mosquito control pathways based on the olfactory system.

\section{Conclusions}

In this study, we performed a systematic comparative analysis of the expression of OR genes in $A e$. aegypti mosquitoes, $\mathrm{A}$ large number of ORs with significant intergroup differences and high expression levels were found.OR88, OR107, OR114,OR115-V2 and OR115-V3 differed significantly between males and females and may have the function of regulating host localization,OR115-V2, OR115-V3, OR116 are down-regulated after blood-feeding and may be involved in regulating the blood-sucking behavior,OR27, OR30, OR62,OR86,OR91, OR117 are up-regulated after blood-feeding and may be involved in regulating the post-sucking search for egg-laying sites, OR27, OR62, OR91, OR75, OR77, OR110 may be involved in regulating the egg-laying behavior.Some of these genes are reported for the first time, providing possible targets for the development of mosquito control based on the olfactory system.

\section{Declarations}

\section{Ethics approval and consent to participate}

Not applicable.

\section{Consent for publication}

All authors have reviewed and approved this manuscript for publication.

\section{Availability of data and materials}

The datasets supporting the conclusions of this article are included within the article.

\section{Competing interests}


The authors declare that they have no competing interests.

\section{Funding}

This work was funded by grants from the National Natural Science Foundation of China (No. 81371847).

\section{Author contributions}

$\mathrm{CXL}, \mathrm{MN}$ and TZ designed the experiments and drafted the manuscript. MN and TZ performed the experiments and conducted the data analysis. HXL, MJL, DX and TYZ conducted a feasibility analysis on the rationality of the experimental design. CXL drafted the final version of the manuscript. All authors read and approved the final manuscript.

\section{References}

1. Bhatt S, Gething PW, Brady OJ, Messina JP, Farlow AW, Moyes CL, et al. The global distribution and burden of dengue. Nature. 2013;496(7446):504-507.

2. McMeniman CJ, Corfas RA, Matthews BJ, Ritchie SA, Vosshall LB. Multimodal integration of carbon dioxide and other sensory cues drives mosquito attraction to humans. Cell. 2014;156(5):1060-1071.

3. Liu H, Xie L, Cheng P, Xu J, Huang $X$, Wang $H$, et al. Trends in insecticide resistance in Culex pipiens pallens over 20 years in Shandong, China. Parasit Vectors. 2019;12(1):167.

4. Liu H, Liu L, Cheng P, Yang L, Chen J, Lu Y, et al. Bionomics and insecticide resistance of Aedes albopictus in Shandong, a high latitude and high-risk dengue transmission area in China. Parasit Vectors. 2020;13(1):11.

5. Hallem EA, Carlson JR. Coding of odors by a receptor repertoire. Cell. 2006;125(1):143-160.

6. Clyne PJ, Warr CG, Freeman MR, Lessing D, Kim J, Carlson JR. A novel family of divergent seventransmembrane proteins: candidate odorant receptors in Drosophila. Neuron. 1999;22(2):327-338.

7. Vosshall LB, Amrein H, Morozov PS, Rzhetsky A, Axel R. A spatial map of olfactory receptor expression in the Drosophila antenna. Cell. 1999;96(5):725-736.

8. Hill CA, Fox AN, Pitts RJ, Kent LB, Tan PL, Chrystal MA, et al. G protein-coupled receptors in Anopheles gambiae. Science. 2002;298(5591):176-178.

9. Bohbot J, Pitts RJ, Kwon HW, Rützler M, Robertson HM, Zwiebel LJ. Molecular characterization of the Aedes aegypti odorant receptor gene family. Insect Mol Biol. 2007;16(5):525-537.

10. Arensburger P, Megy K, Waterhouse RM, Abrudan J, Amedeo P, Antelo B, et al. Sequencing of Culex quinquefasciatus establishes a platform for mosquito comparative genomics. Science. 2010;330(6000):86-88.

11. Chen XG, Jiang X, Gu J, Xu M, Wu Y, Deng Y, et al. Genome sequence of the Asian Tiger mosquito, Aedes albopictus, reveals insights into its biology, genetics, and evolution. Proc Natl Acad Sci U S A. 2015;112(44):E5907-E5915. 
12. Wheelwright $M$, Whittle CR, Riabinina O. Olfactory systems across mosquito species. Cell Tissue Res. 2021;383(1):75-90.

13. Carey AF, Carlson JR. Insect olfaction from model systems to disease control. Proc Natl Acad Sci U S A. 2011;108(32):12987-12995.

14. Krieger J, Klink O, Mohl C, Raming K, Breer H. A candidate olfactory receptor subtype highly conserved across different insect orders. J Comp Physiol A Neuroethol Sens Neural Behav Physiol. 2003;189(7):519-526.

15. Jones WD, Nguyen TA, Kloss B, Lee KJ, Vosshall LB. Functional conservation of an insect odorant receptor gene across 250 million years of evolution. Curr Biol. 2005;15(4):R119-R121.

16. Rinker DC, Zhou X, Pitts RJ; AGC Consortium, Rokas A, Zwiebel LJ. Antennal transcriptome profiles of anopheline mosquitoes reveal human host olfactory specialization in Anopheles gambiae. BMC Genomics. 2013;14:749.

17. Carey AF, Wang G, Su CY, Zwiebel LJ, Carlson JR. Odorant reception in the malaria mosquito Anopheles gambiae. Nature. 2010;464(7285):66-71.

18. Wang G, Carey AF, Carlson JR, Zwiebel LJ. Molecular basis of odor coding in the malaria vector mosquito Anopheles gambiae. Proc Natl Acad Sci U S A. 2010;107(9):4418-4423.

19. Hallem EA, Nicole Fox A, Zwiebel LJ, Carlson JR. Olfaction: mosquito receptor for human-sweat odorant. Nature. 2004;427(6971):212-213.

20. McBride CS, Baier F, Omondi AB, Spitzer SA, Lutomiah J, Sang R, et al. Evolution of mosquito preference for humans linked to an odorant receptor. Nature. 2014;515(7526):222-227.

21. Bohbot JD, Jones PL, Wang G, Pitts RJ, Pask GM, Zwiebel LJ. Conservation of indole responsive odorant receptors in mosquitoes reveals an ancient olfactory trait. Chem Senses. 2011;36(2):149160.

22. Bohbot JD, Dickens JC. Characterization of an enantioselective odorant receptor in the yellow fever mosquito Aedes aegypti. PLoS One. 2009;4(9):e7032.

23. Jung JW, Baeck SJ, Perumalsamy H, Hansson BS, Ahn YJ, Kwon HW. A novel olfactory pathway is essential for fast and efficient blood-feeding in mosquitoes. Sci Rep. 2015;5:13444.

24. latrou K, Biessmann H. Sex-biased expression of odorant receptors in antennae and palps of the African malaria vector Anopheles gambiae. Insect Biochem Mol Biol. 2008;38(2):268-274.

25. Matthews BJ, McBride CS, DeGennaro M, Despo O, Vosshall LB. The neurotranscriptome of the Aedes aegypti mosquito. BMC Genomics. 2016;17:32.

26. Das De T, Thomas T, Verma S, Singla D, Chauhan C, Srivastava V, et al. A Synergistic Transcriptional Regulation of Olfactory Genes Drives Blood-Feeding Associated Complex Behavioral Responses in the Mosquito Anopheles culicifacies. Front Physiol. 2018;9:577.

27. Rinker DC, Pitts RJ, Zhou X, Suh E, Rokas A, Zwiebel LJ. Blood meal-induced changes to antennal transcriptome profiles reveal shifts in odor sensitivities in Anopheles gambiae. Proc Natl Acad Sci U S A. 2013;110(20):8260-8265. 
28. Chen Q, Pei D, Li J, Jing C, Wu W, Man Y. The antenna transcriptome changes in mosquito Anopheles sinensis, pre- and post- blood meal. PLoS One. 2017;12(7):e0181399.

29. Wu Q, Li CX, Liu QM, Guo XX, Shi QM, Zhang HD, et al. RNA interference of odorant receptor CquiOR114/117 affects blood-feeding behavior in Culex quinquefasciatus. Acta Trop. 2020;204:105343.

30. Leal WS, Barbosa RM, Xu W, Ishida Y, Syed Z, Latte N, et al. Reverse and conventional chemical ecology approaches for the development of oviposition attractants for Culex mosquitoes. PLoS One. 2008;3(8):e3045.

31. Pelletier J, Hughes DT, Luetje CW, Leal WS. An odorant receptor from the southern house mosquito Culex pipiens quinquefasciatus sensitive to oviposition attractants. PLoS One. 2010;5(4):e10090.

32. Liu H, Liu T, Xie L, Wang X, Deng Y, Chen CH, et al. Functional analysis of Orco and odorant receptors in odor recognition in Aedes albopictus. Parasit Vectors. 2016;9(1):363.

33. Hughes DT, Pelletier J, Luetje CW, Leal WS. Odorant receptor from the southern house mosquito narrowly tuned to the oviposition attractant skatole. J Chem Ecol. 2010;36(8):797-800.

34. Liu H, Liu L, Cheng P, Huang X, Gong M. An odorant receptor from Anopheles sinensis in China is sensitive to oviposition attractants. Malar J. 2018;17(1):348.

\section{Figures}

\section{Figure 1}

Differences in antennal odorant receptor gene expression between males and females of Ae. aegypti 


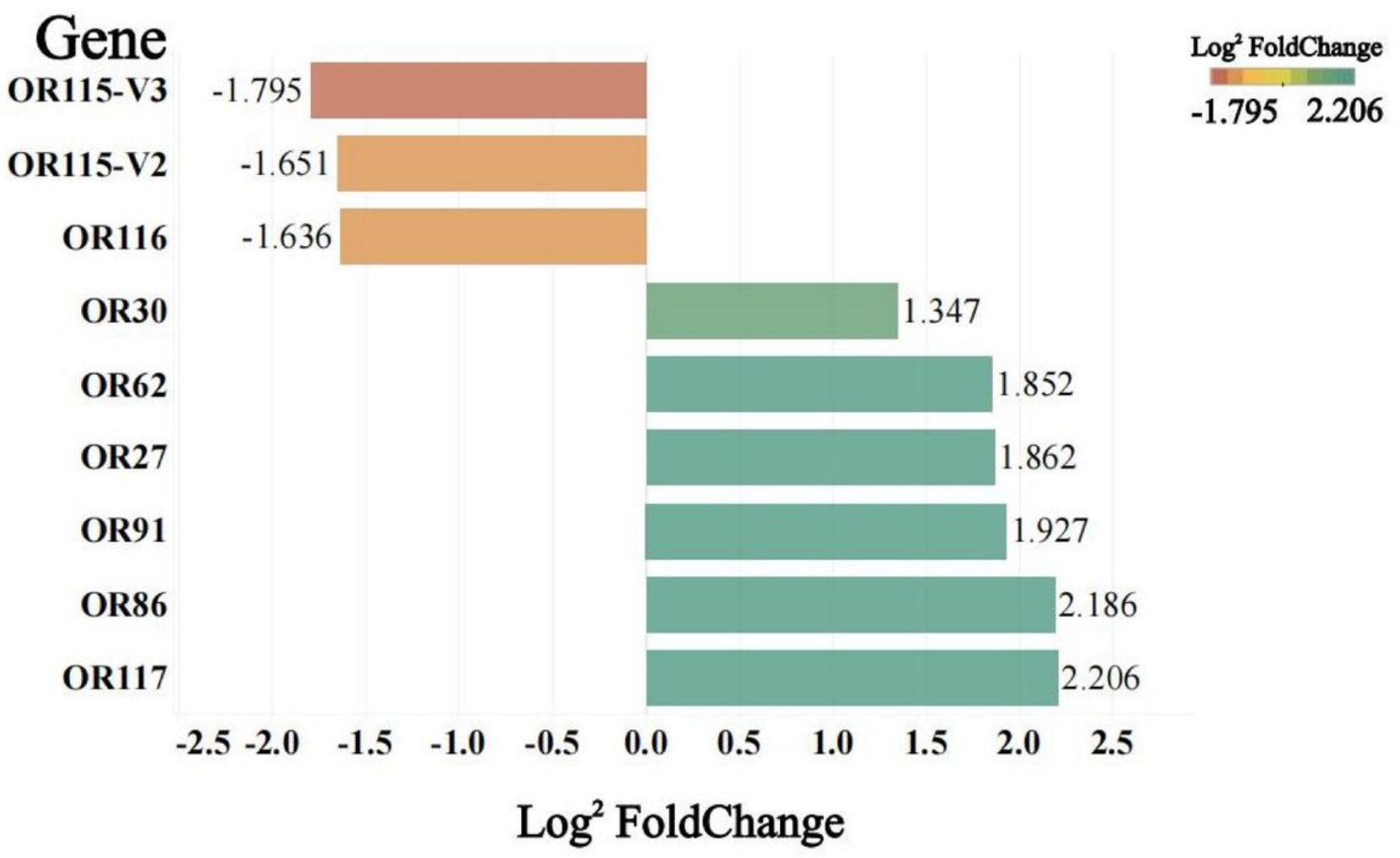

Figure 2

Differences in antennal odorant receptor gene expression between pre-versus post-blood-feeding females of Ae. aegypti 


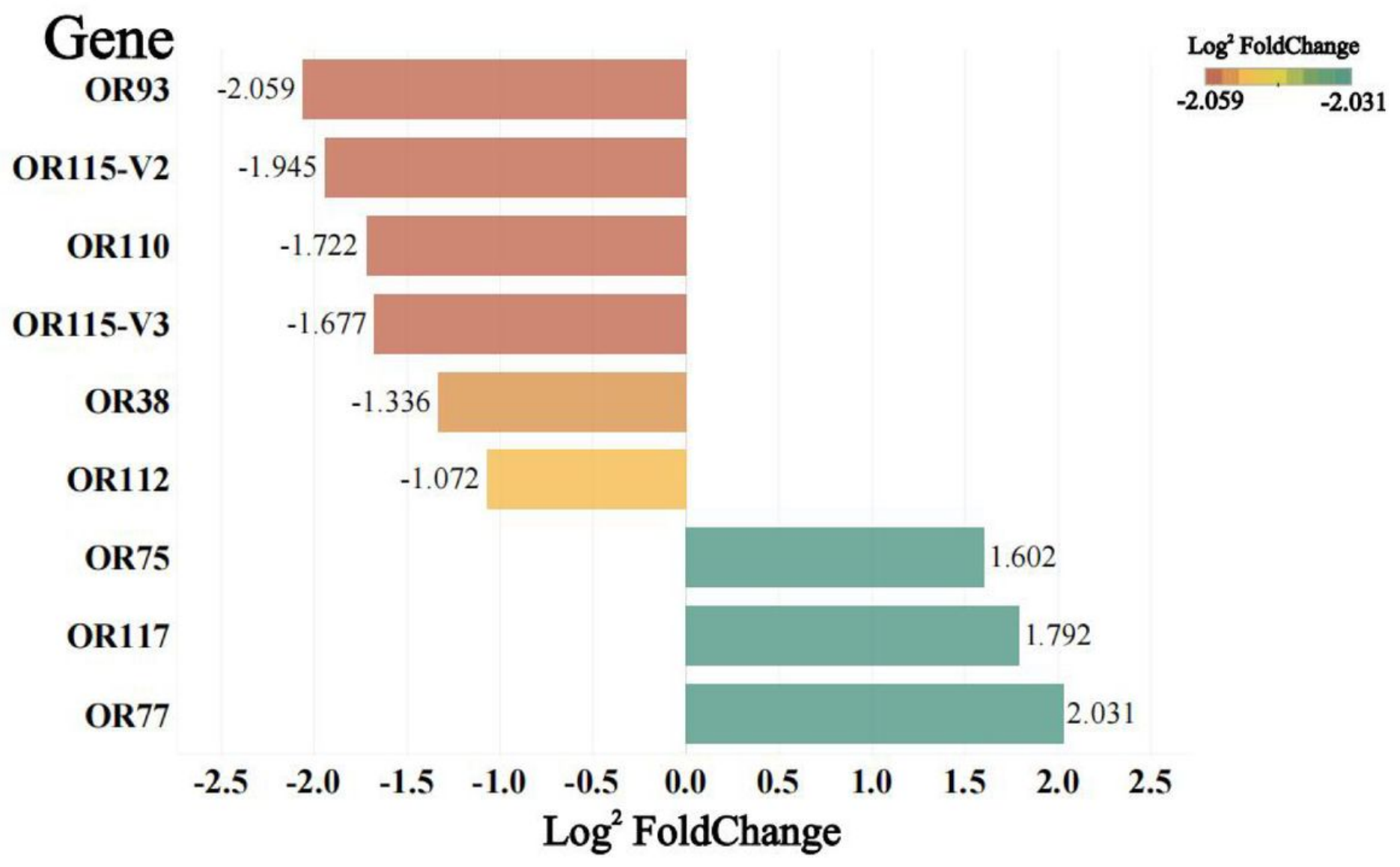

Figure 3

Differences in antennal odorant receptor gene expression between pre-versus post-oviposition females of Ae. aegypti 
Gene

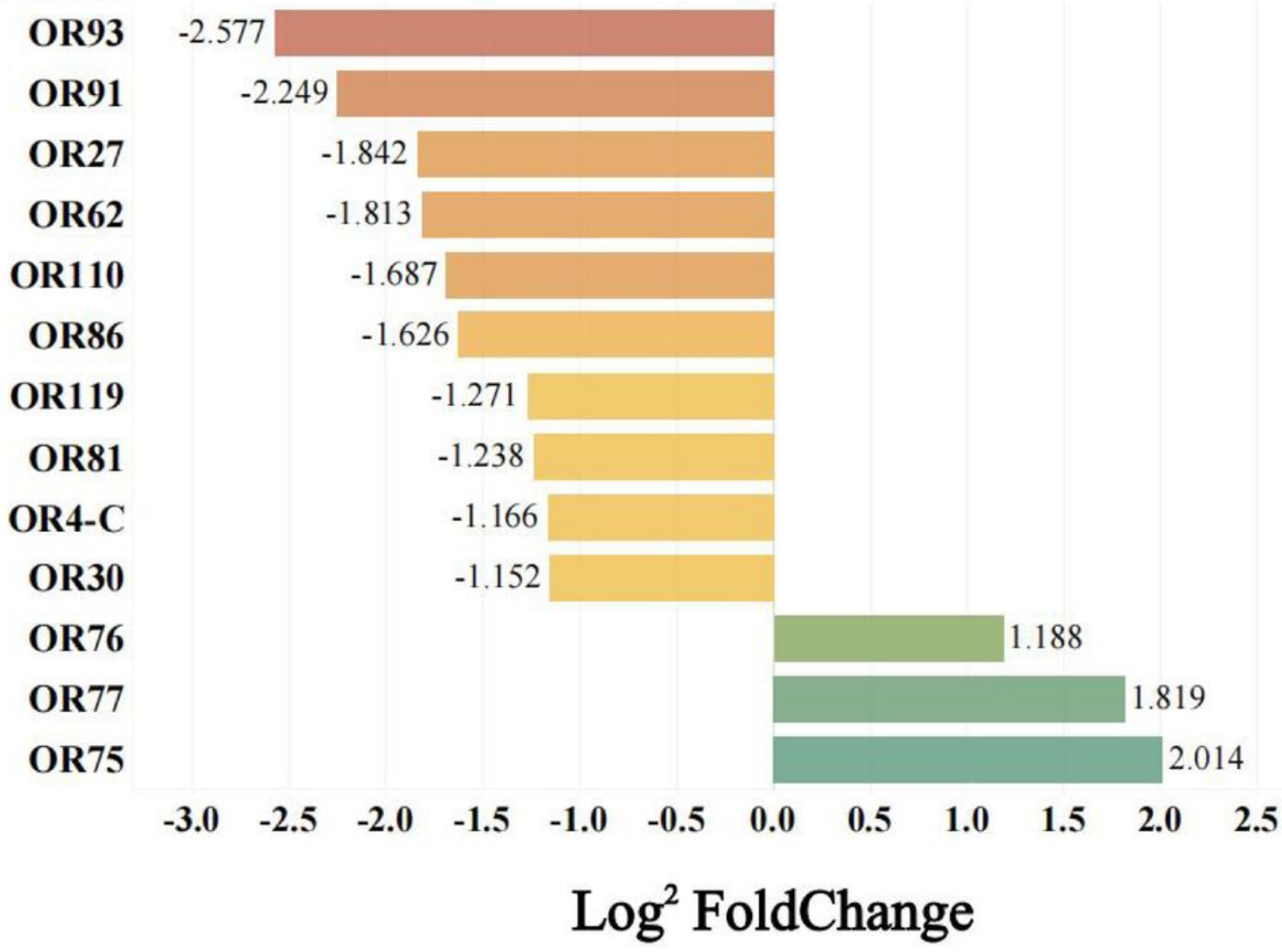

$\log ^{2}$ FoldChange

$-2.577 \quad 2.014$

Figure 4

Differences in antennal odorant receptor gene expression between post-blood-feeding females versus post-oviposition females of Ae. aegypti 

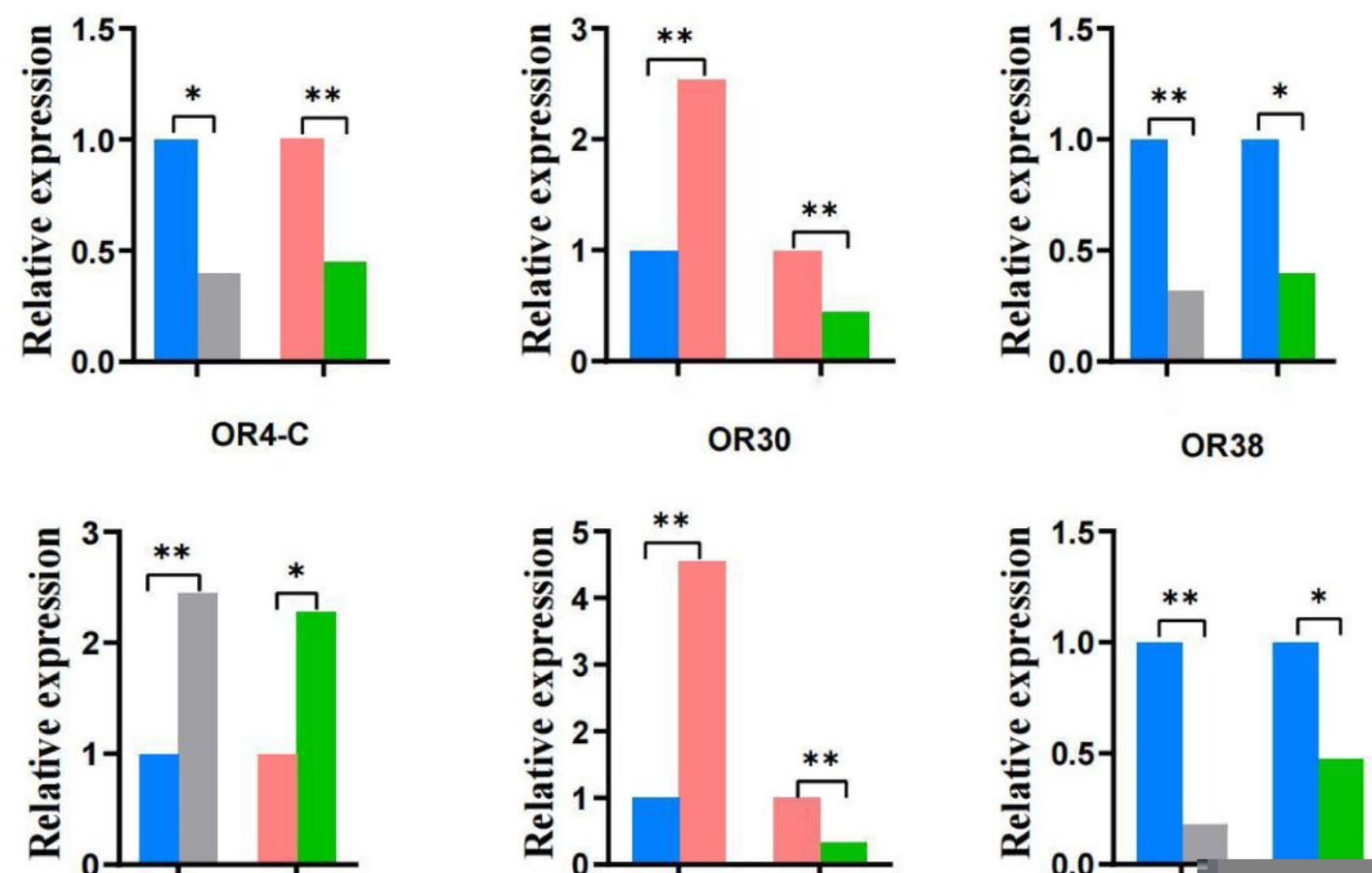

Figure 5

Two of the four groups of comparisons reveal differentially expressed Ae. aegypti antennae odorant receptor genes. * indicates $\mathrm{P}<0.05$, $* \star$ indicates $\mathrm{P}<0.01$. 


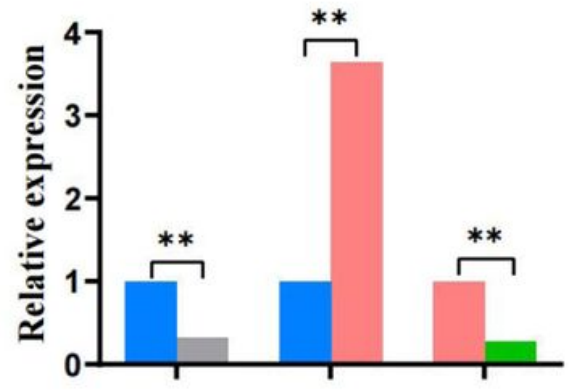

OR27

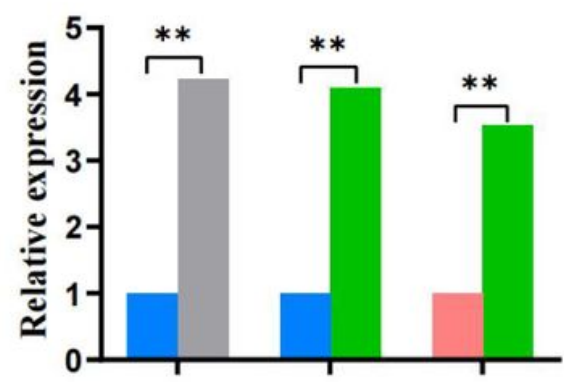

OR77

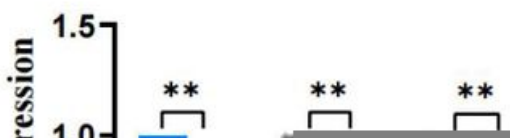

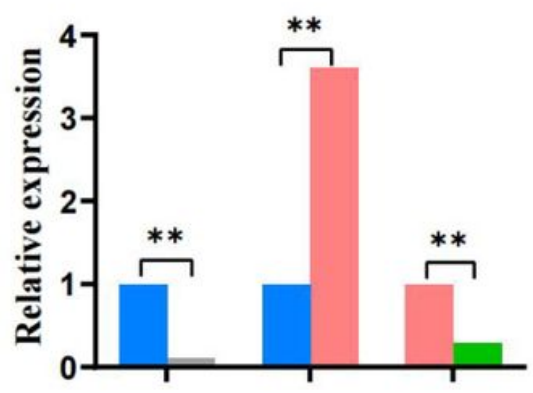

OR62
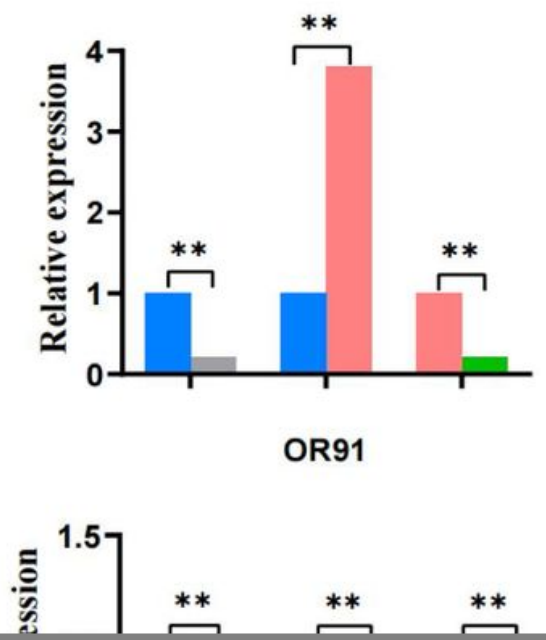

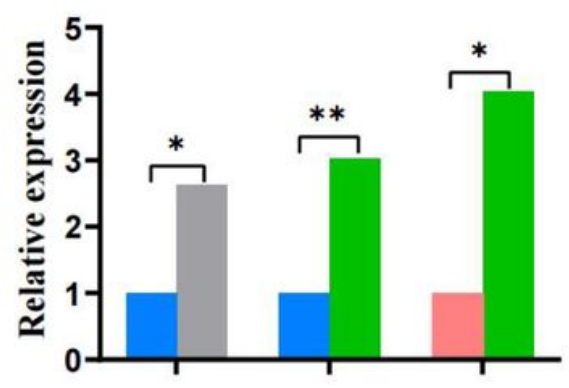

OR75

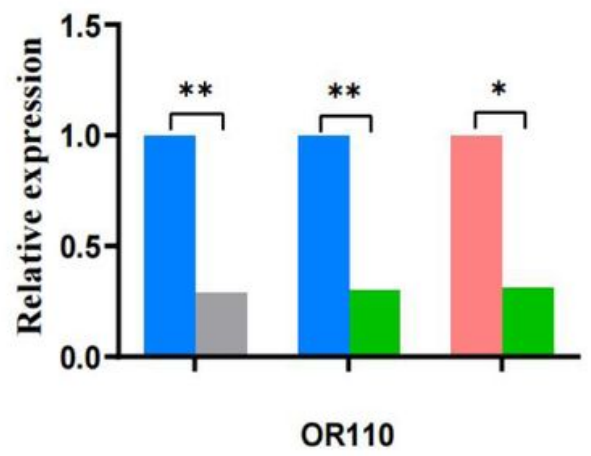

Enmaln

Figure 6

Three of the four groups of comparisons reveal differentially expressed Ae. aegypti antennae odorant receptor genes.

\section{Supplementary Files}

This is a list of supplementary files associated with this preprint. Click to download.

- Additionalfile1DatasetS1.docx

- Additionalfile2DatasetS2.docx

- graphicalabstract.pdf 